\title{
Dark Matter Annihilation Feedback in Cosmological Simulations II: The Influence on Gas and Halo Structure
}

\author{
N. Iwanus ${ }^{1 \star}$, P. J. Elahi ${ }^{2,3}$, F. List ${ }^{1}$ and G. F. Lewis ${ }^{1}$ \\ ${ }^{1}$ Sydney Institute for Astronomy, School of Physics, A28, The University of Sydney, NSW 2006, Australia \\ ${ }^{2}$ International Centre for Radio Astronomy Research, University of Western Australia, 35 Stirling Highway, Crawley, WA 6009, Australia \\ ${ }^{3}$ ARC Centre of Excellence for All Sky Astrophysics in 3 Dimensions (ASTRO 3D)
}

Accepted XXX. Received YYY; in original form ZZZ

\begin{abstract}
We present new cosmological hydrodynamic simulations that incorporate Dark Matter Annihilation Feedback (DMAF), whereby energy released from the annihilation of dark matter particles through decay channels such as photon or positron-electron pairs provide additional heating sources for local baryonic material. For annihilation rates comparable to WIMP-like particles, we find that the key influence of DMAF is to inhibit gas accretion onto halos. Such diminished gas accretion early in the lifetimes of halos results in reduced gas fractions in smaller halos, and the delayed halo formation times of larger structures, suggesting that DMAF could impact the stellar age distribution in galaxies, and morphology of dwarfs. For a dark matter particle mass of $m_{\chi} \sim 10 \mathrm{MeV}$, there is a 'critical halo mass' of $\sim 10^{13} \mathrm{M}_{\odot}$ at $z=0$, below which there are large differences when compared to $\Lambda \mathrm{CDM}$, such as a reduction in the abundance of halo structures as large as 25 percent, reduced gas content by 50 percent and central gas densities reduced down to 10 percent within halos of mass $\sim 10^{12} \mathrm{M}_{\odot}$ but with increasing effects in smaller halos. Higher dark matter particle mass models have a smaller 'critical halo mass'. For a $m_{\chi} \sim 100 \mathrm{MeV}$ model, we find differences start appearing below halo masses of $\sim 10^{12} \mathrm{M}_{\odot}$ and a $m_{\chi} \gtrsim 1 \mathrm{GeV}$ model, this mass scale lies below the resolution of our simulations, though we still observe changes in the morphology of dwarf galaxies.
\end{abstract}

Key words: (cosmology:) large-scale structure of Universe, (cosmology:) dark matter, galaxies: formation

\section{INTRODUCTION}

The standard cosmological model, where the Universe is dominated by dark energy and cold dark matter, has been shown to provide excellent agreement with observations of the larger-scale cosmos (e.g. Planck Collaboration et al. 2016). However, predictions from high-resolution numerical simulations based on $\Lambda \mathrm{CDM}$ cosmologies, appear to be in tension with the smaller-scale structure of the Universe (Bullock \& Boylan-Kolchin 2017). For example, numerical dark matter halos possess sharp central cusps in their density profiles, while rotation curves of nearby dwarf galaxies indicate that they reside in cored halos (Kravtsov et al. 1998; Oh et al. 2015). Furthermore, numerical universes appear to overproduce satellites around Milky Way-like galaxies (Klypin et al. 1999; Moore et al. 1999), an issue that could be solved with baryon feedback effects like UV radiation (Thoul \& Weinberg 1996; Barkana \& Loeb 1999; Bullock et al. 2000). However a baryon feedback solution leads to another form of tension, the existence of halos that are seemingly 'too-big-to-fail' to produce galaxies (Boylan-Kolchin et al. 2011; Griffen et al. 2016).
With the advent of the first high-resolution hydrodynamical simulations in which the stellar components of galaxies could be resolved, it became apparent that realistic galaxy formation required additional heating, such as stellar feedback, to prevent overcooling (Katz \& Gunn 1991; Ceverino \& Klypin 2009; Hopkins et al. 2012; Rey-Raposo et al. 2017). The sources of this energy injection, include star formation, supernovae, black holes and feedback from active galactic nuclei (AGN), represent physical processes on scales, often not resolved in cosmological simulations (Somerville \& Davé 2015). Large dynamical scales are required to resolve such feedback processes and large scale structure simultaneously, which significantly stretches computational resources, forcing a compromise between volume and resolution. Typically small-scale processes such as star formation, are treated with socalled 'sub-grid' methods. Sub-grid methods incorporate simplified models that aim to capture the essential physics of these phenomena while relaxing the need for resolution. For example, calculating the details of a single supernovae explosion is extremely computationally expensive, and is, in itself an outstanding astrophysical problem. Yet supernovae feedback is essential in regulating stellar formation in galaxies (Ceverino \& Klypin 2009; Rey-Raposo et al.

^ E-mail: nikolas.iwanus@sydney.edu.au 
2017), and can possibly induce core formation in dark matter halos (Pontzen \& Governato 2012).

Feedback mechanisms from baryonic physics are widely proposed to offer solutions to the small scale problems of $\Lambda \mathrm{CDM}$, although, unfortunately, it has been found that the effect of these sub-grid models of the same physical process can vary between different implementations and resolution (Sembolini et al. 2016a,b; Elahi et al. 2016; Cui et al. 2016; Arthur et al. 2017). For example, different simulation groups investigating core formation in isolated dwarf halos find contradictory results with each other, some finding cored dwarf halos 'all the way down' (Read et al. 2016), no cores at all (Fattahi et al. 2016), or core formation is suppressed at certain scales (Tollet et al. 2016; Fitts et al. 2017). However, recent simulations have shown that some of the problems of $\Lambda \mathrm{CDM}$, such as too-big-to-fail, can be curtailed with more modern codes and stateof-the-art baryon and feedback models (Sawala et al. 2016; Wetzel et al. 2016).

While there has been significant focus upon feedback from baryonic processes, the possibility of processes originating from the 'dark sector', such as self-interacting dark matter (SIDM), fuzzy dark matter (FDM), warm dark matter (WDM) and annihilating dark matter, have been neglected in simulations in the past. If present, however, such feedback will have an impact on structure formation and evolution. For example, self-interacting dark matter (van den Aarssen et al. 2012) has been proposed as a complement to baryon physics in alleviating some of the small scale discrepancies (Spergel \& Steinhardt 2000). In high density environments, a weak self-scattering cross section can cause dark matter to experience pressure like forces, resulting in thermalised cores in the inner regions of halos. At the same time SIDM acts more like collisionless CDM in the low density field, where scattering events are rare, thus replicating the same large scale predictions. In recent years a wide range of studies utilising $\mathrm{N}$-body implementations of SIDM have converged upon cross sections $\sigma / m \approx 0.1-10 \mathrm{~cm}^{2} \mathrm{~g}^{-1}$ in dwarf galaxies that are consistent with observations of small structure (Valli \& Yu 2018; Ren et al. 2018), but also leave large scale structure predictions of $\Lambda \mathrm{CDM}$ intact. Separating core formation by SIDM or baryonic means remains an active area of research (Vogelsberger et al. 2014; Fry et al. 2015; Dooley et al. 2016; Kamada et al. 2017; Tulin \& Yu 2018; Robertson et al. 2018). Recently Robles et al. (2017) have found that SIDM can produce cores in small dwarfs with stellar masses $M_{\star}<10^{6} M_{\odot}$. At these scales core formation that would be typically be induced by stellar feedback is cut off by UV photo-ionisation.

Fuzzy dark matter models, such as ultra-light axions (Hu et al. 2000), have also been proposed as models where their physics can leave marks on the Universes' small scales. New N-body simulations that feature these have recently been produced and their implications are being investigated (Veltmaat \& Niemeyer 2016; Veltmaat et al. 2018; Du et al. 2018; Nori \& Baldi 2018).

Theoretically a dark matter candidate called Weakly Interacting Massive Particles (WIMPs), self-annihilate in the early Universe and provide an explanation for the dark matter abundance observed today (i.e $\Omega_{m} \approx 0.3$ ), assuming an early universe cross section $\langle\sigma v\rangle=3 \times 10^{-26} \mathrm{~cm}^{3} \mathrm{~s}^{-1}$ (Steigman et al. 2012). Many such particles have been independently hypothesised in particle physics to solve different theoretical problems in the Standard Model, and so are seen as viable candidates for these thermal relics (Feng 2010). As such, significant effort has been directed towards observing the annihilation/decay signals produced from various astrophysical sources containing high dark matter densities such as the Galactic Centre and dwarf spheroidal galaxies. In recent years, a number of studies in conjunction with CMB measurements have constrained the annihilation rate to levels nearing thermal relic cross section, for models where the dark matter particle mass is less than $60 \mathrm{GeV}$ and decay into single standard model channels, ruling out those simple thermal relic WIMPs models (Ackermann et al. 2014, 2015; Albert et al. 2017). Ground based experiments also place limits at $\mathrm{TeV}$ ranges, although not yet near the thermal cross-section (Profumo et al. 2016; Oakes et al. 2017; Rinchiuso et al. 2017). Relaxing some of the simplifying, albeit restrictive, assumptions about WIMP annihilation results in weaker constraints, for example, multiple decay channels, or the presence of $2 \rightarrow 3$ channels, result in softened annihilation spectra which are more difficult to detect. Simply allowing decay into a combination of the well studied single $2 \rightarrow 2$ channels, weakens the constraints such that we cannot yet rule out WIMPs as light as 2-6 GeV in simple generic thermal dark matter (Leane et al. 2018). Multi-stepped cascade decays within the dark sector can weaken Gamma ray limits by 1-2 orders above the thermal rate for particles as light as $\sim 1$ $\mathrm{GeV}$, dependent on the number of cascades and standard model final states (Elor et al. 2016), and the presence of $3 \rightarrow 2$ 'Cannibal dark matter' interactions are relatively unconstrained in the velocity dependant case (Pappadopulo et al. 2016). When cross sections vary with velocity, a generic feature of many models, the cross section limits from the Fermi Gamma-ray telescope are severely weakened as the astrophysical $\mathrm{J}$ factors depend on the velocity distribution functions of matter, and weaken cross section constraints by as much as 3 orders of magnitude, above the thermal rate (Zhao et al. 2016, 2018). Other model complications exist as well, but more complications generally only adds more uncertainty to the crosssection constraints. Taken in aggregate this means that the case for $\mathrm{GeV}$ WIMP dark matter is yet not as pessimistic as sometimes implied, see Leane et al. (2018) for a discussion on the case for $\mathrm{GeV}$ WIMPs.

Below $\approx 1 \mathrm{GeV}$ in the $\mathrm{MeV}$ ranges, the current strongest limits on s-wave annihilation arise from the era of recombination, at $\mathrm{z} \approx$ 1100, via imprints on CMB (Planck Collaboration et al. 2016)

$\frac{f_{e f f}\langle\sigma v\rangle}{m_{\chi}}<4.1 \times 10^{-31} \mathrm{~cm}^{3} \mathrm{~s}^{-1} \mathrm{MeV}^{-1}$,

where $f_{\text {eff }}<1$ is a parameter that relates the energy released that can effectively increase the ionisation fraction, thus strongly restricting the simple s-wave thermal WIMP scenario in the sub-GeV range. A number of missions like e-ASTROGAM and AMEGO will be able to probe the the sub-GeV annihilation products directly today with Fermi Gamma-ray telescope like sensitivity (Bartels et al. 2017). Voyager I direct measurements of cosmic ray data however are able to rule out thermal relic WIMPs below about 300 $\mathrm{MeV}$ today (Boudaud et al. 2017) assuming single decay product annihilations and models of Galactic cosmic ray propagation. Sub$\mathrm{GeV}$ dark matter could arise in non-standard thermal production mechanisms or suppression of the cross section at early times (Choquette et al. 2016; Xiang et al. 2017) or non-WIMP models. Like the higher energy Gamma ray detection experiments, minor extensions to the simple WIMP scenario can drastically alter the constraints, for example p-wave annihilation at the sub-GeV ranges is much less constrained by the CMB and well above the thermal cross sections (Diamanti et al. 2014). While the WIMP scenario provides a convenient picture of dark matter production from thermal freeze out, it is not the only dark matter model and it is unclear how the previous limits would apply to generic dark matter models. The theoretical uncertainties in the dark sector and the nature of 
dark matter make it difficult to rule out anything but simple models or specific candidates.

Another less explored avenue of investigation into dark matter annihilation raises the question if any energy released by the decay and subsequent interaction of the annihilation products could play an active role in late structure-formation (Ascasibar 2007). The expected interaction products in the form of particles and photons deposit their energy in to the nearby baryonic material of galaxies and the intergalactic medium, and a number of studies show that Dark Matter Annihilation Feedback (DMAF) could affect gas flows, cosmic rays and early star formation (Ripamonti et al. 2007; Natarajan et al. 2008; Wechakama \& Ascasibar 2011; Schön et al. 2015, 2018).

To fully understand the impact from DMAF we require numerical simulations to properly capture the non-linear structure growth and to date there has not yet been a self-consistent, hydrodynamic, cosmological simulation including the effects of DMAF from generic dark matter models. In Iwanus et al. (2017, hereafter Paper I) we presented the self-consistent hydrodynamic code that includes the effect of DMAF (which we briefly review in 2) and tested in the case of isolated toy halo models. In this second paper we present the first cosmological simulations containing DMAF, evolved from perturbations at $\mathrm{z}=100$ and report on the differences brought on by DMAF compared to non-cooling simulations focusing on the properties of halos. This paper runs as follows, in section 2 we briefly review our DMAF code implementation and our halo finding code and describe the set up of our simulation boxes. In section 3 we investigate changes to the simulations in the particle properties, halo catalogues and halo profiles and conclude our study in section 4 .

\section{METHODS}

\subsection{DMAF Implementation}

Full details of the code implementation into GADGET-2 are provided in paper I, but here we briefly give an outline. We utilise the standard SPH kernel to estimate the density of dark matter surrounding a gas particle,

$\rho_{i}=\sum_{j=1}^{N} M_{j} W\left(r_{i j}, h_{i}\right)$,

where $M_{j}$ is the mass of a nearby dark matter particle, $W$ the smoothing kernel, $r_{i j}$ is the particle separation and $h_{i}$ the smoothing length. The DMAF specific power released by the gas particles in our simulation is

$\frac{d u_{i}}{d t}=\frac{\langle\sigma v\rangle c^{2}}{m_{\chi}} \frac{\rho_{\chi}^{2}}{\rho_{g}}$,

where $\langle\sigma v\rangle$ is the thermal cross section, $c$ is the speed of light, $m_{\chi}$ is the dark matter particle mass, $\rho_{g}$ is the particles baryonic density and $\rho_{\chi}$ is the estimated dark matter density as seen locally by the gas particle. To account for dark matter particles annihilating away DM particles can experience mass loss given by

$$
\frac{d M_{i}}{d t}=-\frac{\langle\sigma v\rangle}{m_{\chi}} \rho_{\chi} M_{i}
$$

where $M_{i}$ is the mass of the N-body dark matter particle and here $\rho_{\chi}$ is the local dark matter density ${ }^{1}$ (,see Paper I and Monaghan 1992, for more details). We assume the mean energy deposition length of the particles is contained within the SPH smoothing length i.e., all energy injection is localised.

We initialise our simulations using N-GenIC (Grossi \& Springel 2009; Springel 2015), though a fully self-consistent simulation should also include the effects of DMAF in the early Universe. However we are primarily focused on the non-linear regime to explore DMAF on late forming structures and so we ignore the effect of DMAF on the initial conditions, but they could result in increased abundances of dwarf halos at mass scales of about $\approx$ $\mathrm{M}_{\odot}$ (Bertschinger 2006), well below the resolutions we study here. More complicated models like late-decoupling scenarios with additional SIDM might affect the power spectrum and affect structure at dwarf scales, similar to warm dark matter (Bringmann et al. 2016; Binder et al. 2016). It should be noted that in general, the thermally averaged cross section in the early universe is not necessarily the same during late time structure formation. Generic models dark matter models have velocity and spatial dependencies on the particle environments which will differ for late and early annihilation, but in this work we restrict ourselves to a single constant velocity cross section.

\subsection{Halo Catalogues: VELOCIRAPTOR \& TREEFROG}

We generated our halo catalogues using VELOCIRAPTOR, formerly STRUCTURE-FINDER (Elahi et al. 2011, Elahi et al., in prep $)^{2}$. The code identifies candidate halos first with a 3D friendsof-friends (3DFOF) algorithm, and then applies a 6DFOF algorithm to cleanse the catalogue and separate halos connected by spurious particle bridges. Substructures within the field objects are identified by first identifying particles that appear to be dynamically distinct from the background halo particles, i.e. particles which have a local velocity distribution that differs significantly from the mean. These outlier particles are then linked using a phase-space FOF. This approach is capable of not only finding subhaloes, but also tidal streams surrounding subhaloes as well as tidal streams from completely disrupted subhaloes (Elahi et al. 2013). For this analysis, we are only interested in subhaloes, i.e., self-bound objects, and thus require particles in substructures to have potential energy to kinetic energy ratios of at least 0.95 .

Linking the structures between different catalogues in time we used the code TREEFROG which is part of the VELOCIRAPTOR package. At its core, TREEFroG matches two objects at different times based on a merit function of the number of shared unique particle IDs between each simulation (see Srisawat et al. 2013; Elahi et al. 2018, and Elahi in prep., for more details).

\subsection{The Simulations}

Our simulations consist of $100 h^{-1} \mathrm{Mpc}$ co-moving length periodic boxes, containing $\mathrm{N}=512^{3} \mathrm{~N}$-body gas and dark matter particles (see Table 1). Our simulation volume was chosen to resolve

\footnotetext{
1 Although we found this effect to be negligible for the cross sections used in this work, in paper I and so was turned off after finding negligible differences in low resolution simulations. It may still be relevant in future work with hidden dark sector models.

2 Open source code available at https://github.com/pelahi/VELOCIraptorSTF
} 


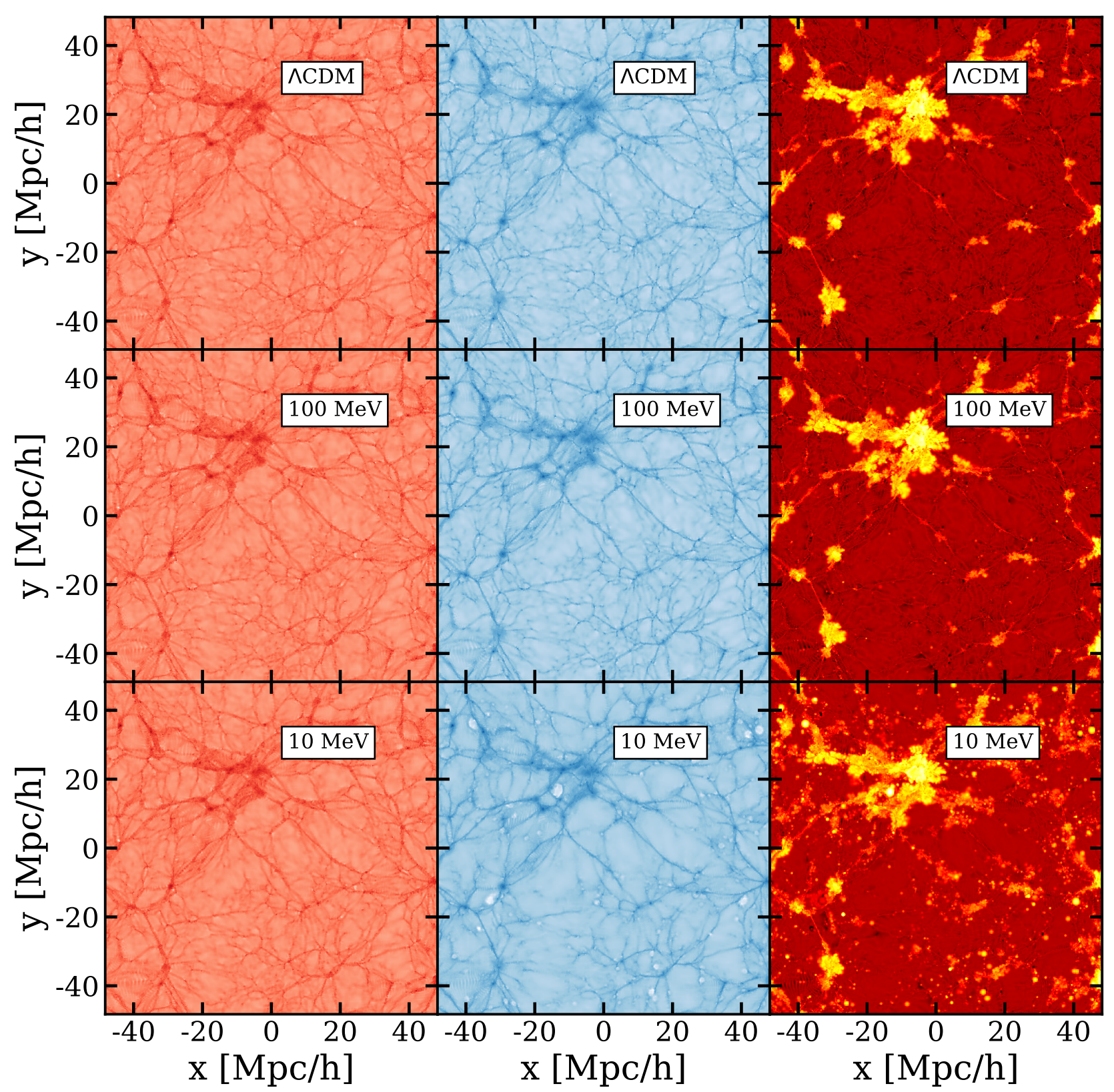

Figure 1. A 2D-Kernel SPH image of some of our simulations. Each row shows a slice of a $\Lambda$ CDM control simulation (top row) as well as simulations with an annihilating dark matter model with $\mathrm{m}_{\chi}=100 \mathrm{MeV}$ (middle) and an a stronger $10 \mathrm{MeV}$ model (bottom) all with the thermal relic cross section a standard thermal cross section $\langle\sigma v\rangle=3 \times 10^{-26}$. The left column images (red) show the dark matter density, middle (blue) shows the gas density and thermal energy is shown on the right columns (yellow/red). The $100 \mathrm{MeV}$ model has only impacted strongly the small scale structure making differences hard to see, however the $10 \mathrm{MeV}$ model now effects structures large enough to be seen and results in a much smoother field with less filamentary structure.

smaller halos as our previous study and tests in paper 1 indicated that they were more susceptible to the effects of DMAF — largely due to their decreasing binding energies and higher concentrations. Smaller volumes however reduce the amount of cosmic variance of a simulation, but can also affect the properties of small halo populations due to reduction in tidal interactions by larger clusters. We chose $100 h^{-1} \mathrm{Mpc}$ as a compromise between size and resolution. These parameters at the highest resolutions, allowed us to find bound halos as small as about $10^{10} h^{-1} \mathrm{M}_{\odot}$.

The same initial conditions were rerun multiple times under different dark matter models with varying mass and a correspond- ing control simulation of a pure non-cooling hydrodynamics (no stars, AGN etc.), allowing us to directly track any differences due to DMAF. Our cosmological parameters are taken from the Planck collaboration (Planck Collaboration et al. 2016). For ease of comparison to the rest of literature, which is dominated by thermal WIMP astrophysical signatures, we have set a constant relic-like cross section and varied the particles mass (see Table 2). However, with our efficient and localised energy injection scheme, see equation 3, decreasing the mass is equivalent to raising the cross section, so the models here should be considered more general than the thermal relic WIMPs. For example, a $100 \mathrm{GeV}$ model can also 

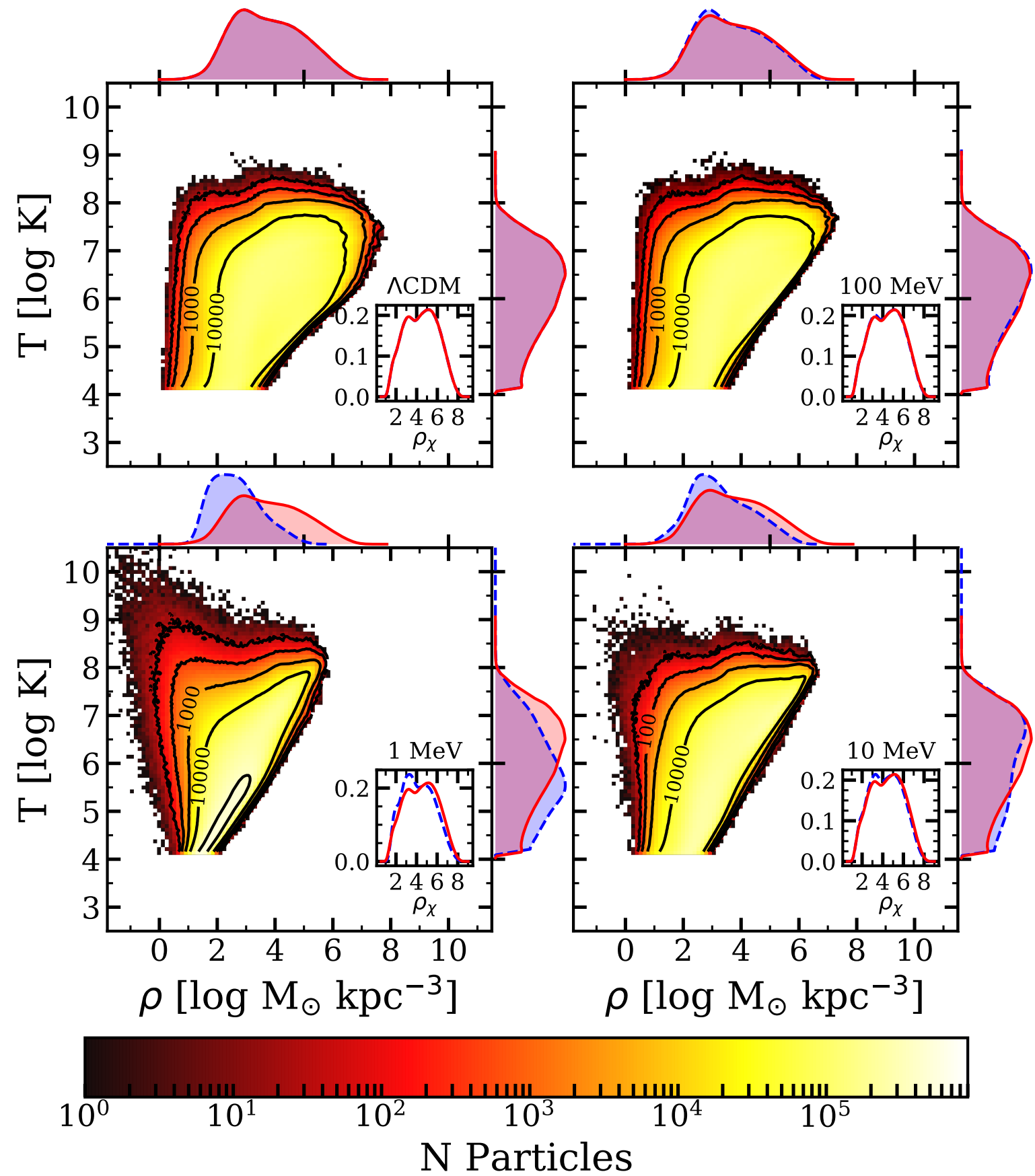

Figure 2. The 2D Histogram showing the temperature and density phase space distribution of the gas in the $\Lambda \mathrm{CDM}, 100 \mathrm{MeV}, 10 \mathrm{MeV}$ and $1 \mathrm{MeV}$ simulations (clockwise). We show the marginal pdfs of the gas density (top add-ons) and the temperature (right add-ons) in in dashed lines compared to the $\Lambda$ CDM case (solid line). For clarity in the figures we have shown the distribution for only the particles with $\mathrm{T}>10^{4}$, our minimum temperature floor. The insets similarly show the corresponding distribution of dark matter particle densities (blue) and comparison with the $\Lambda$ CDM DM density distribution (red).

represent a standard s-wave annihilation with mass of $1 \mathrm{TeV}$ and at a higher cross section of $\langle\sigma v\rangle=3 \times 10^{-25} \mathrm{~cm}^{3} \mathrm{~s}^{-1}$ which are not yet strongly constrained even for single species, s-wave annihilation.

We note that although there are strong constraints on light $~$ sub $\mathrm{MeV}$ dark matter models in the simple WIMP paradigm, it is by no means the only one, and the effects of a generic annihilating dark matter species injecting energy at sites of high $\rho_{\chi}^{2}$ during structure formation should be thoroughly understood in exploratory
$\mathrm{N}$-body simulations before the study of more complex models with p-wave or exotic velocity dependence, multiple-species DM, coannihilation etc., much like SIDM studies have progressed into studying non-constant cross sections. In future work, we will generalise our DMAF scheme to study more complex dark matter models with sub-dominant components, non-constant cross sections, late forming dark matter, etc. For this reason we choose to study DMAF for a number of dark matter masses in the standard WIMP thermal freeze-out scenario, including masses ruled out by the $\mathrm{CMB}$ 
Table 1. The GADGET-2 simulation settings used for our largest resolution simulation.

\begin{tabular}{lc}
\hline Simulation Settings & Value \\
\hline Box length & $100 h^{-1} \mathrm{Mpc}$ \\
Particles N & $2 \times 512^{3}$ \\
DM N-body mass & $5.38 \times 10^{8} h^{-1} \mathrm{M}_{\odot}$ \\
Gas N-body mass & $0.848 \times 10^{8} h^{-1} \mathrm{M}_{\odot}$ \\
$\epsilon_{\text {soft }}$ & $9.76 h^{-1} \mathrm{kpc}$ \\
$\mathrm{N}_{\text {sph }}$ & 40 \\
$\mathrm{~T}_{\text {min }}$ & $10^{4} \mathrm{~K}$ \\
$\mathrm{z}_{\text {init }}$ & 100 \\
\hline
\end{tabular}

Table 2. Our cosmological parameters are taken from the the Planck 2015 measurements (Planck Collaboration et al. 2016)

\begin{tabular}{lc}
\hline Cosmological Parameter & Value \\
\hline$\Omega_{\Lambda}$ & 0.6911 \\
$\Omega_{m}$ & 0.3075 \\
$\Omega_{b}$ & 0.0486 \\
$\sigma_{8}$ & 0.8159 \\
$h$ & 0.6774 \\
$n_{s}$ & 0.9667 \\
$\langle\sigma v\rangle$ & $3 \times 10^{-26} \mathrm{~cm}^{3} \mathrm{~s}^{-1}$ \\
$m_{\chi}$ & $100 \mathrm{keV}, 1 \mathrm{MeV}, 10 \mathrm{MeV}$, \\
& $100 \mathrm{MeV}, 1 \mathrm{GeV}, 100 \mathrm{GeV}$ \\
\hline
\end{tabular}

(Planck Collaboration et al. 2016), specifically the $100 \mathrm{keV}$ model. This extreme heating model provides a 'stress-test' of the code, allowing us to identify numerical limitations of the scheme as well as aiding in the interpretation of other models. Additionally, such low masses are not definitively ruled out in population suppressed or non-standard production models as in Heeck \& Teresi (2017) and Berlin \& Blinov (2018).

\section{RESULTS}

We show the matter distribution in Figure 1, where the red and blue panels show a slice of the smoothed density fields of dark matter and gas respectively, while the last yellow-red column shows the specific thermal energy. At the scales presented here, there is little perceptible difference between the $100 \mathrm{MeV}$ simulation and the $\Lambda \mathrm{CDM}$ control simulation, showing that the large scale structure is seemingly unaltered for moderate heating levels, though we will see smaller scale effects later. Ramping up the the annihilation rate by decreasing the dark matter particle mass to $m_{\chi}=10 \mathrm{MeV}$ visibly affects the distribution, smoothing out much of the smaller filamentary structures. For the models with even stronger annihilation, large DM overdensities appear to lack gas. As we further increase the rates of annihilation with $1 \mathrm{MeV}$ and $100 \mathrm{keV}$, we see large bubbles of hot low density gas begin to form, some of which are also visible to a lesser degree in the $m_{\chi}=10 \mathrm{MeV}$ simulation. These bubbles form in regions where high density dark matter structures inject large amounts of thermal energy into low density gaseous regions.

These bubbles form even in simulations using smaller and global time stepping schemes. They are not a result of time-step numeric artifacts that can be diminished by using careful timestepping methods as addressed by Saitoh \& Makino (2009). Instead these form due to our assumption that the gas is always efficiently and locally capturing the DMAF energy, even in low density gas. A simple check was performed by repeating lower resolution runs but now adding a simple switch where DMAF heating is turned off in gas particles where densities are lower than the cosmic mean; see Appendix A. This strongly suppressed the growth of these bubbles and the following results that appear in the rest of this paper were found to be insensitive to the time-stepping method as well as the DMAF cutoff switch, thus do not affect the conclusions in the following sections. This is because the bubbles form in areas of low density hot gas, making them gravitationally insignificant and unbound to the halos structures within our simulations; again, see Appendix A.

\subsection{Particle properties}

In Figure 2 we show the temperature and density phase space distribution of the gas particles at $\mathrm{z}=0$, where we calculated the temperature of a particle as

$T=\frac{\mu m_{p}(\gamma-1)}{k_{B}} u$

and we assumed the standard GADGET-2 settings of mean molecular mass of $\mu=1.22, \gamma=\frac{5}{3}, m_{p}$ is the mass of a proton, $\mathrm{k}_{B}$ and $u$ is the specific thermal energy of the particle. We find that the particles in high density environments have been preferentially heated due to annihilation feedback, as can be seen by the straightening of the lower right edge of the distribution in the $10 \mathrm{MeV}$ case and a smaller change in the $100 \mathrm{MeV}$ case. Overall however, although there is a slight increase in gas particle numbers at high temperatures above $10^{8} \mathrm{~K}$, there is a much larger deficiency of particles at temperatures between $10^{6}$ and $10^{8} \mathrm{~K}$ and a large increase in the number of colder gas particles below $10^{6} \mathrm{~K}$. The apparent net effect of annihilation feedback is to prevent condensation and to expel gas particles from high density environments where now fewer but hotter gas particles generate enough pressure to push against the infalling gas which remains cold. In the cases with annihilation stronger than $10 \mathrm{MeV}$, we see little or no gas exists at high densities as DMAF was strong enough to prevent any gas condensation into the dark matter halos. The insets of each panel in Figure 2 show the corresponding PDFs of the dark matter particle densities. They are only slightly affected in the $100 \mathrm{MeV}$ simulation but show a decrease in the number of dark matter particles at high densities in the 10 and $1 \mathrm{MeV}$ simulation, suggesting that gas changes due to DMAF have gravitationally imprinted onto the dark matter as well.

\subsection{Halo Populations}

We quantify the mass of our halos using the common virial radius definition such that,

$M_{200}=200 \rho_{c} \frac{4 \pi}{3} R_{200}^{3}$,

that is the mass enclosed within a radius, $R_{200}$ that contains an average density 200 times greater than the critical density of the universe, $\rho_{c}$. The maximum circular velocity is calculated as

$V_{\max }^{2}=\max \left(\frac{\mathrm{GM}(\mathrm{r})}{\mathrm{r}}\right)$,

where $M(r)$ is the enclosed mass within radius $r$. 


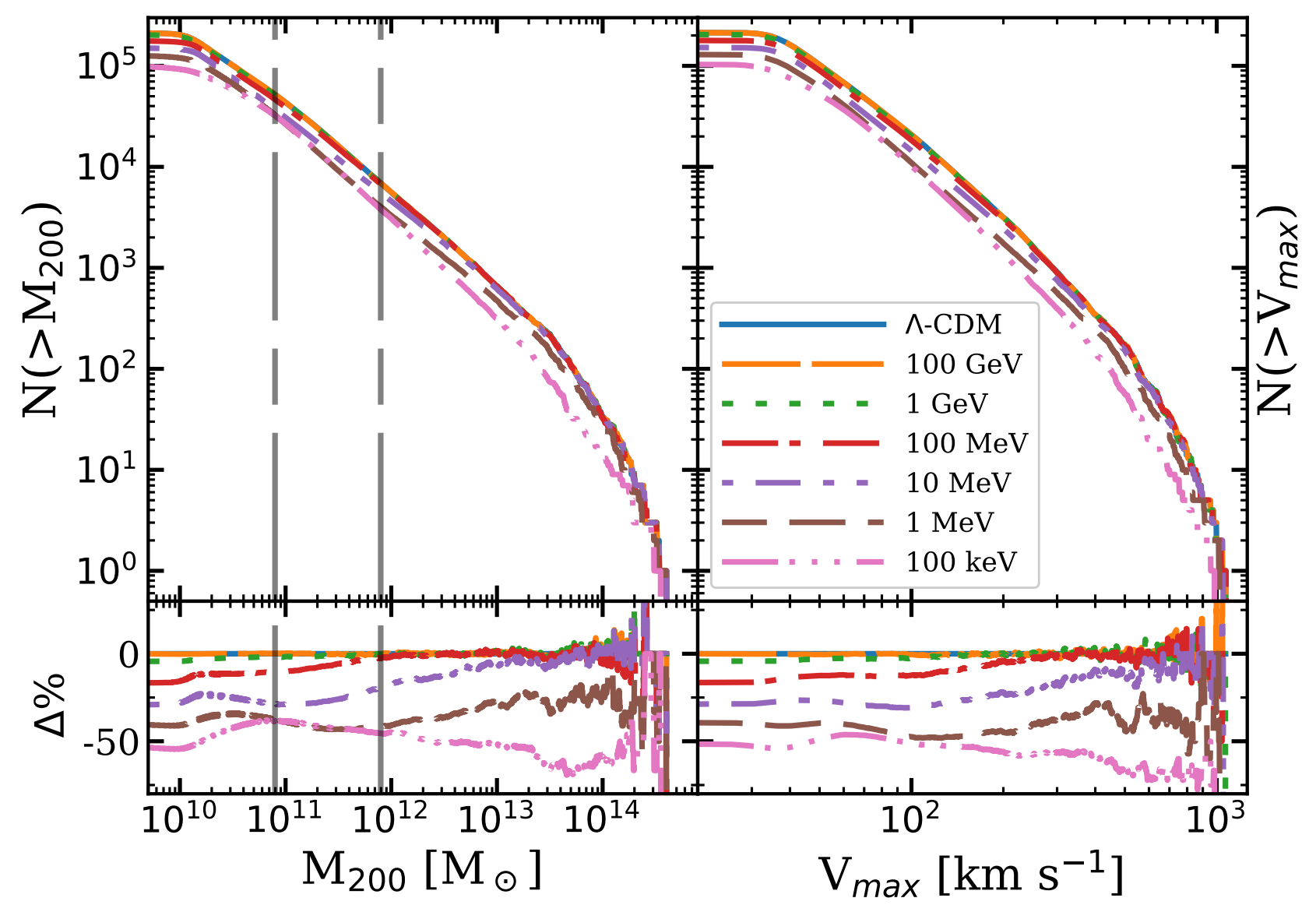

Figure 3. The Halo Mass Function (left) at redshift $z=0$ and the Halo Velocity Function (right) of our simulations. The residual plots show the percentage difference in the HMF/HVF compared to the control $\Lambda \mathrm{CDM}$ simulation. For the middle annihilation rate $\left(\mathrm{m}_{\chi}=100 \mathrm{MeV}\right)$, DMAF tends to lead to suppression of halo abundance at lower masses, as gas accretion has been prevented in smaller concentrated halos, delaying their growth. Halos with masses $>10^{13} \mathbf{M}_{\odot}$ do not show reduction in abundance except for the strong annihilation models with $\mathrm{m}_{\mathcal{\chi}}=100 \mathrm{keV}$ and $\mathrm{m}_{\mathcal{\chi}}=1 \mathrm{MeV}$, where the annihilation is drastic enough as to slow the growth of even the large halos. The $\mathrm{m}_{\chi}=100 \mathrm{MeV}$ model shows a reduction in abundances for halos below $10^{12} \mathrm{M}_{\odot}$, and we see that the $\mathrm{m}_{\chi}=1$ $\mathrm{GeV}$ model is starting to show sensitivity to DMAF.

We show the Halo Mass Function (HMF) and Halo Velocity Function (HVF) in Figure 3 as well as the associated deviation from the control simulation in the bottom panels. During convergence testing, (see Figure 4), comparison with lower resolution simulations showed little deviation from their higher resolution counterparts when looking at masses above about 1000 dark matter particles (dashed vertical line), and only minor differences near 100 particles for all simulations. In Paper I and other studies (Ripamonti et al. 2007; Schön et al. 2015, 2018) we had concluded that small concentrated halos tended to be more sensitive to the effects of DMAF due to their high concentrations and lower gravitational binding energy. However the residuals for our strongest feedback model still show large deficits in high mass halo abundance. The $100 \mathrm{GeV}$ model shows little change overall whilst the $1 \mathrm{GeV}$ and $100 \mathrm{MeV}$ models show agreement with our conclusions from Paper I, that the abundance of low mass objects is suppressed ${ }^{3}$. The onset of this abundance reduction increases with stronger annihilation,

3 Although it does begin to rise up again below $\mathrm{M}_{200} \approx 10^{11} \mathrm{M}_{\odot}$ when the halos only consist of $\approx 100$ particles for some of the models. occurring just below a 'critical halo mass' near $10^{12} \mathrm{M}_{\odot}$ for $m_{\chi}=$ $100 \mathrm{MeV}, 10^{13} \mathrm{M}_{\odot}$ for $m_{\chi}=10 \mathrm{MeV}$ and shows reduction at all scales for the strongest models $m_{\chi}=1 \mathrm{MeV}$ and $m_{\chi}=100 \mathrm{keV}$, the critical mass being above our largest halo mass.

Figure 5 sheds some light on the origin of these differences by showing the evolution of the HMF through time. For $\mathrm{m}_{\chi}=$ $100 \mathrm{MeV}$, the differences were larger at high redshift but decreased with time. In the $m_{\chi}=10 \mathrm{MeV}$ case we see that at early redshifts the abundance is reduced at all scales. The injection of energy and suppression of gas infall has increased the formation time of these structures but for large objects $\left(\mathrm{M}_{200}>10^{13} \mathrm{M}_{\odot}\right)$ the delay does not seemingly prevent the largest halos from reaching their full size and we see only small differences in mass abundances by $z=0$. For smaller objects however, the DMAF induced delay is more enduring and results in the suppression of low mass objects we saw in Figure 3. In the $m_{\chi}=100 \mathrm{keV}$ model this delay is significant enough to have induced a suppression at all scales, only a few halos managing to catching up. It appears that DMAF in the absence of other feedback has introduced new dynamics into structure formation which can be understood by remembering that the primary way small halos grow at early times is due to the accretion of out- 


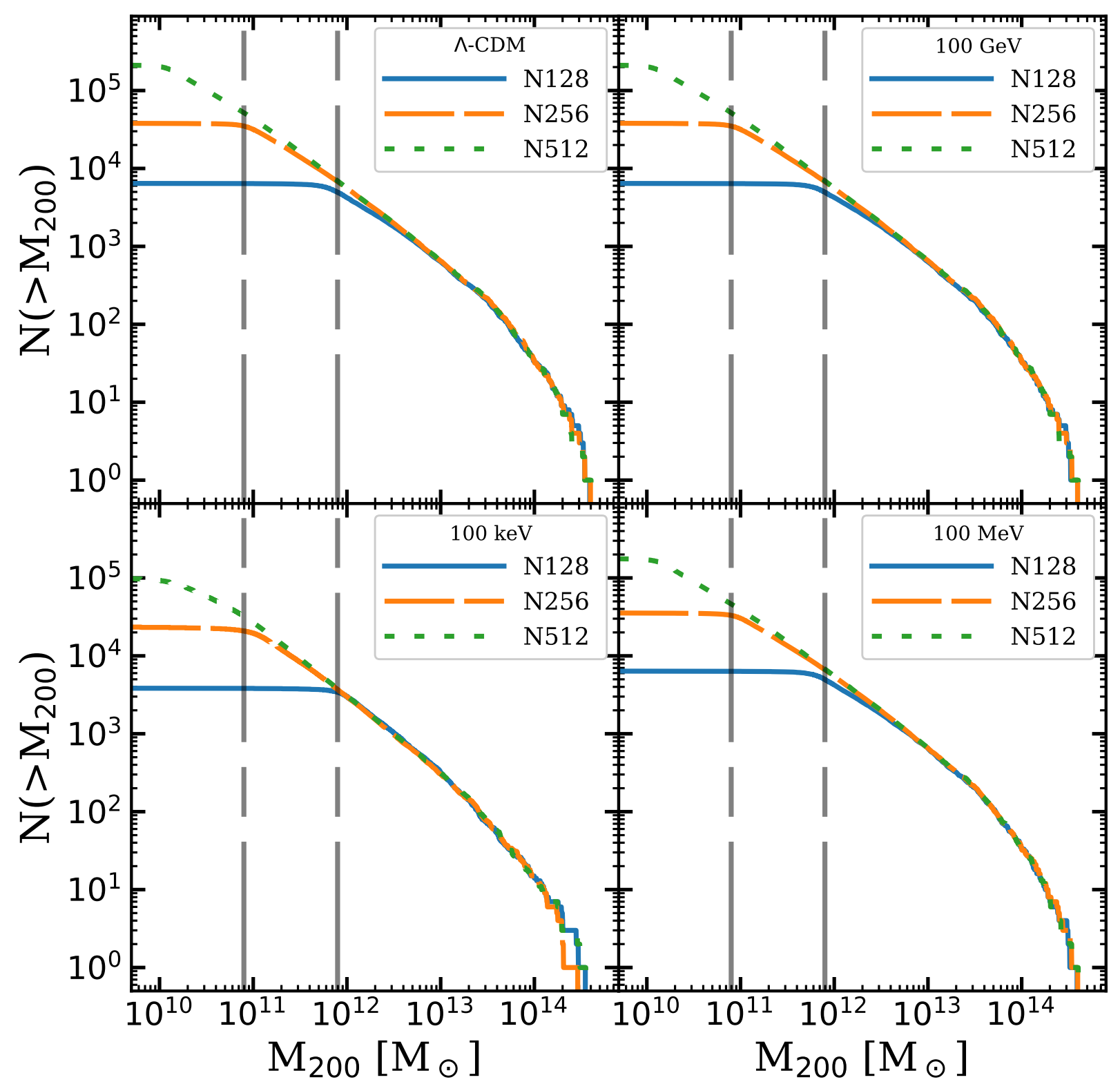

Figure 4. The HMFs for all 4 models sampled at resolutions with $\mathrm{N}=2 \times 128^{3}, 2 \times 256^{3}$ and $2 \times 512^{3}$ particles. Most simulations show good convergence properties in our simulations for all the DMAF models. The grey dashed lines show the mass equivalent of 100 and 1000 dark matter particles respectively in the $512^{3}$ case.

lying material, while the formation of large clusters and galaxies is dominated by the mergers and accretion of smaller halos. DMAF slows the accretion of gas, reducing the growth rate of these small early structures but does little once the large structures begin to grow through mergers. In the $\mathrm{m}_{\chi}=100 \mathrm{keV}$ and $\mathrm{m}_{\chi}=1 \mathrm{MeV}$ simulation we see that DMAF from this extreme model is able to delay the merger growth to such a degree that it does not catch up at the the high mass end.

\subsection{Halo Properties}

The density profiles of halos are reasonably characterised by Navarro-Frenk-White (NFW) halos (Navarro et al. 1996), which follow a profile given by,

$\rho(r)=\frac{\rho_{0}}{\left(\frac{r}{r_{s}}\right)\left(1+\frac{r}{r_{s}}\right)^{2}}$,

where $r_{s}$ is a scale radius, $\rho_{0}$ is a density scale. NFW profiles are often parametrised by their mass, see equation 6 and a concentration parameter given by

$c=\frac{R_{200}}{r_{s}}$.

Concentration parameters in this work are not directly calculated by finding the best fit parameters of the averaged spherical profile. Instead we follow Prada et al. (2012) where we assume an NFW 


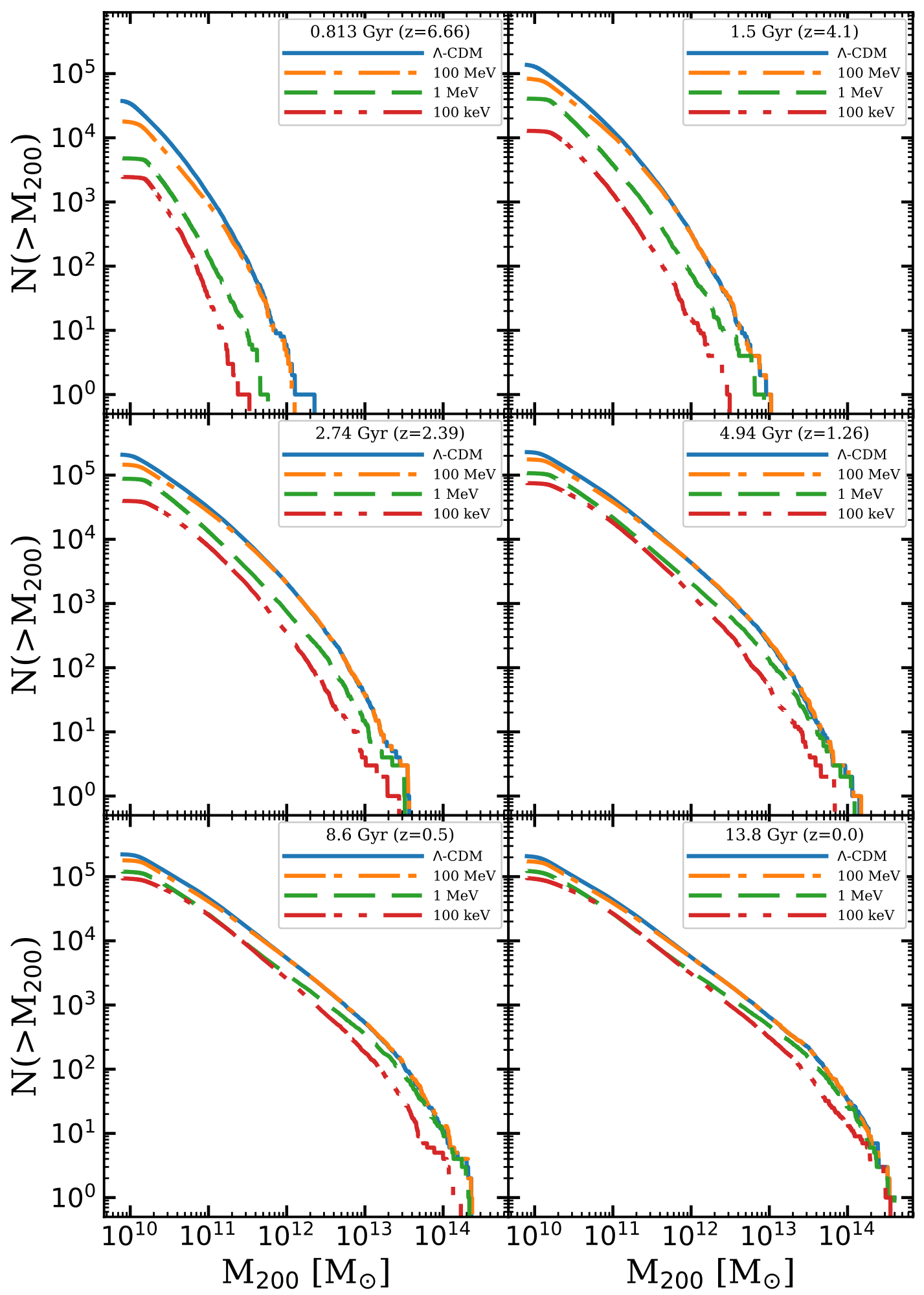

Figure 5. The Halo Mass Function (HMF) time evolution of our 4 simulations. As seen at $z=6.66$ the differences between the DMAF simulations and the control $\Lambda \mathrm{CDM}$ simulation are more pronounced at early times due to DMAF slowing/preventing the accretion of gaseous material into the halos. These differences becomes less pronounced (especially at high masses) as we approach $z=0$ where the largest of the halos in all but in the most extreme $100 \mathrm{keV}$ simulation have negligible mass and abundance differences. This 'catch up' can be understood by remembering that large cluster growth becomes dominated by mergers at late times which DMAF appears to do little to prevent. 


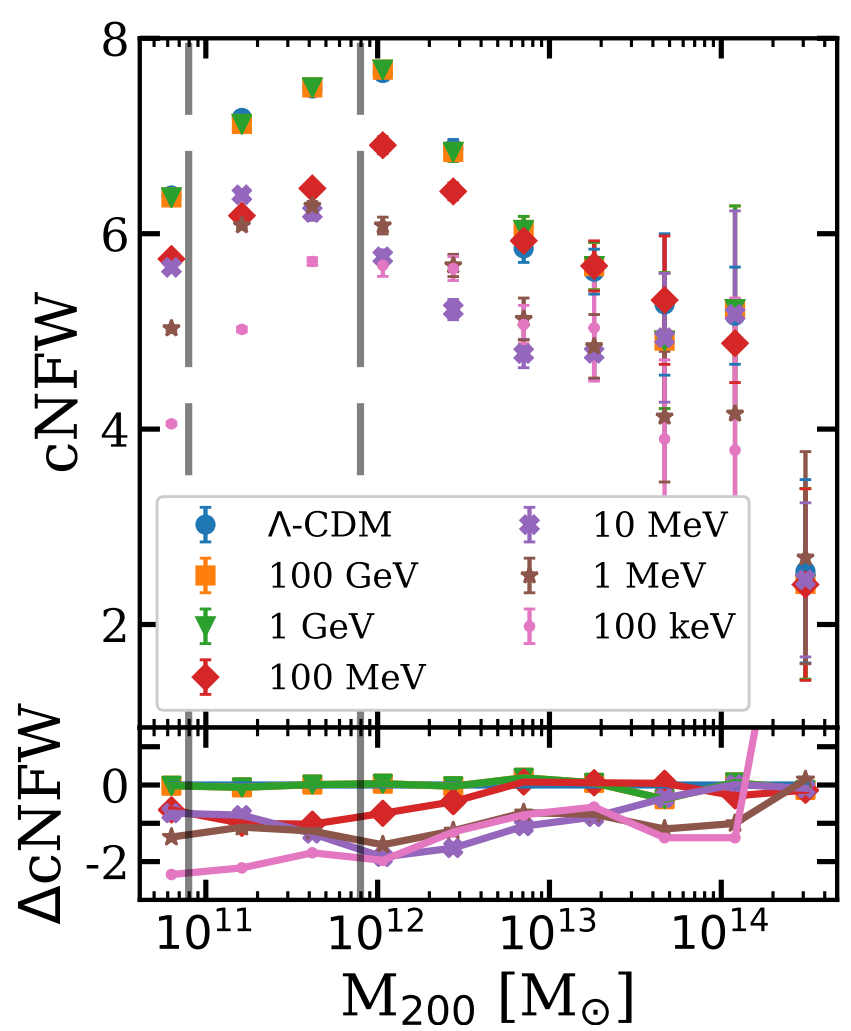

Figure 6. The concentrations of our halos, calculated with equation 10 , under different DMAF models. The error bars are the SEM estimate of each bin and for reference we show two grey dashed lines at a mass equivalent of 100 and 1000 dark matter particle masses and higher masses are better resolved. The lowest panel shows the residual differences of each model compared to the $\Lambda \mathrm{CDM}$ case.

profile, for which the following relation holds.

$$
\frac{V_{\max }^{2}}{G M_{200} / R_{200}}=\frac{0.216 c}{\ln (1+\mathrm{c})-\mathrm{c} /(1+\mathrm{c})} \text {. }
$$

We plot the $c-M$ relation of our halos in Figure 6. The scatter in the distribution is quite large and so we show the standard error of the mean to more clearly show the significance of the shift in the distribution centres. In the stronger feedback models, we see that the concentrations are systematically lower at all masses and the effect is an increase in smaller halos, at least up to the dashed vertical line where the halos are sampled with particle numbers less than 1000 . These changes can occur through two main modes, DMAF directly altering the equilibrium structure of the halos by the increased thermal energy and lower densities in the gaseous component, but also because of the delayed halo formation time, as the c-M relationship is normally a function of redshift as well (Okoli 2017). Early forming structures tend to have higher concentrations as they are able to effectively accrete the outlying materials at larger radii and so the lower concentrations could be attributed partially attributed to delayed halo formation.

In Paper I (Appendix B for details), we showed the importance of resolving the inner core of halos (i.e below $r_{s}$ ) in DMAF simulations as the high density cores are expected to be a major source of annihilation. Cusped halos that are considered unresolved below a scale $r_{c o n}$ will therefore underestimate the total amount of energy injected by the halo by a factor of about $\frac{r_{c o n}}{r_{s}}$ for an NFW halo, $r_{c o n}$ can be determined by the 'Power criterion' (Power et al. 2003). The lack of a resolved cusp in these poorly sampled halos may explain why the trend for the HMF and concentrations reverses at poorly resolved low mass halos.

We investigated the halos spin parameter as defined by Bullock et al. (2001)

$\lambda=\frac{J_{200}}{\sqrt{2} M_{200} V_{200} R_{200}}$,

but we found no clear sign of an increase or decrease brought on by DMAF.

\subsubsection{Gas in Halos}

The gas fraction of our halos is defined as

$f_{b}=\frac{m_{g} N_{g}}{m_{g} N_{g}+m_{\chi} N_{\chi}}$,

where $N_{g}$ and $N_{\chi}$ are the number of gas and DM particles bound to the halo weighted by their N-body particle mass, respectively. We find a strong DMAF signature in the baryonic fraction as shown in Figure 7 relative to the the cosmic baryon fraction $f_{\Omega}=\Omega_{b} / \Omega_{\chi}$. The median fraction is heavily reduced in halos that have masses less than a 'critical mass' the depends on the strength of the annihilation, above which the baryon fraction returns to near normal. In the $1 \mathrm{MeV}$ model only the largest halos at about $\approx 10^{14} \mathrm{M}_{\odot}$ manage to hold onto their gas but drops off to $10^{13} \mathrm{M}_{\odot}$ for the $10 \mathrm{MeV}$ model and just below $10^{12} \mathrm{M}_{\odot}$ for the $100 \mathrm{MeV}$ simulation. We did not see significant gas depletion in the $1 \mathrm{GeV}$ model although given the spacing of the stronger models critical mass it may simply occur just below our simulation resolution. Actually the largest halos seem to be more gas rich in comparison to the $\Lambda$ CDM counterparts, this is likely because there is now more gaseous material available for accretion by the large halos as the small ones were unable to capture their own gas.

In Figure 8 we calculate the average temperature of the gas in each halo and then show the median of these halos in their respective bins. DMAF has reduced the gas reservoirs in small halos below about $10^{12} \mathrm{M}_{\odot}$ of the $100 \mathrm{MeV}$ simulation, and the $100 \mathrm{keV}$ simulation contains essentially no gas for halos with masses below $10^{13} \mathrm{M}_{\odot}$, but what remaining gas is left exists at much larger temperatures. These results are consistent with the picture drawn in section 3.1, where a less dense but higher temperature gas is able to prevent condensation of further accreting gas into the halos.

In comparison to our non-cooling simulations, such changes are likely to be washed out in real life to some degree by cooling and other feedback processes. However the strongest promise of being able to detect these changes is in regions where baryonic feedback simulations have shown that competing cooling and heating processes are of similar magnitudes. At lower masses, dwarf halos become susceptible to the effects of UV photo-ionisation (Hoeft et al. 2006; Fitts et al. 2017) and can prevent the condensation of gas into these dwarf galaxies at a UV heating 'critical mass' of about $10^{10} \mathrm{M}_{\odot}$ as at these scales the cooling and dominant UV heating are near cancelled out. Our results show that even effective $100 \mathrm{MeV}$ models can disrupt the properties of these halos above this mass scale and we see a faint sign of slightly reduced abundances in the HMF and HVF for the $1 \mathrm{GeV}$ model. Though we just fell short of the required resolution in this study, we see it as a promising area for DMAF signatures as even here we see effects of DMAF at Galactic scales. Below this critical mass scale, we expect 


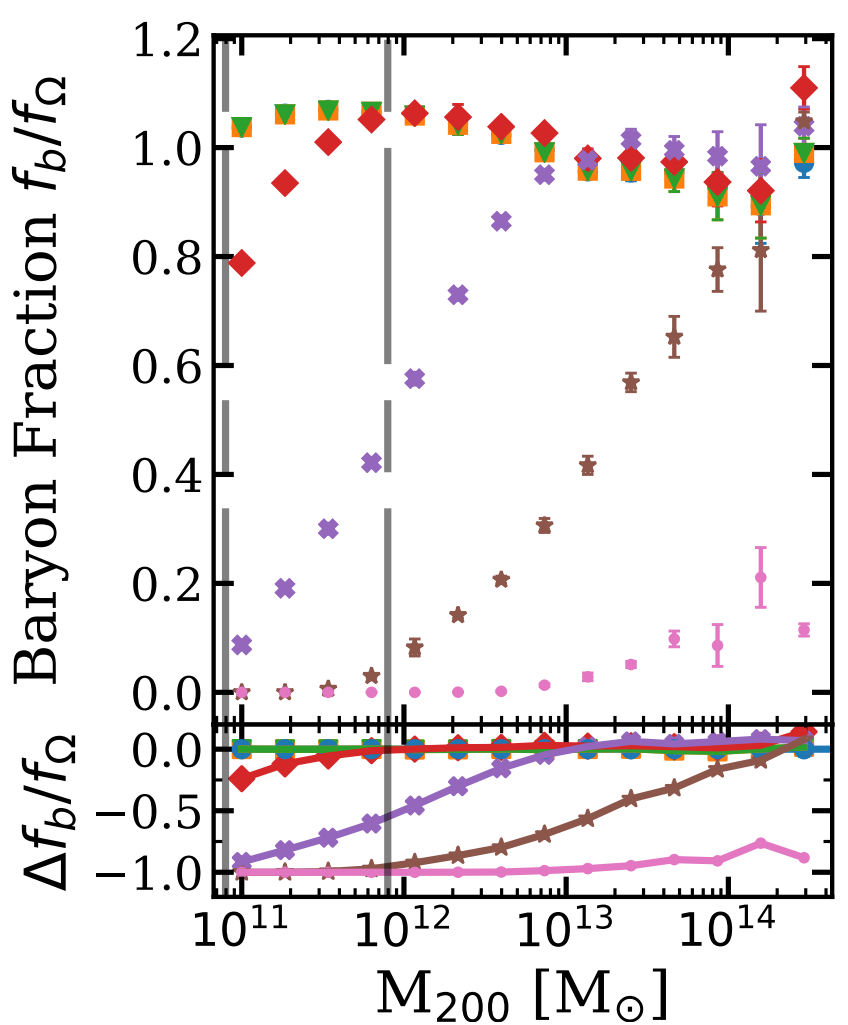

Figure 7. The baryonic fraction, $f_{b}$ relative to the overall cosmic fraction $f_{\Omega}=\Omega_{b} / \Omega_{\chi}$, of the halos at $\mathrm{z}=0$. The error bars show the standard error of the mean in each bin. The fraction is very sensitive to annihilation feedback most strongly at a critical mass of about $10^{13} M_{\odot}$ for the $100 \mathrm{MeV}$ simulation, although the largest halos seem to be more gas rich in comparison to the $\Lambda \mathrm{CDM}$ counterparts, likely because there is now more gaseous material unbound to the smaller objects.

UV heating to be the dominant source of energy, however gas is self-shielding (Krumholz \& Gnedin 2011) which reduces the UV heating efficiency and is taken into account in many modern simulations, whilst an energy source from a dark matter peak could still inject its energy internally bypassing the shielding and producing differences.

\subsection{Halo Profiles}

In Figure 9, we show the averaged gaseous density profile as a function of their scaled radius. We show 4 sub-samples of halos with varying $\mathrm{M}_{200}$ (sample sizes are $N=50,100,100,200$ ) roughly corresponding to our largest clusters, large, medium and small galaxies. The $\Lambda \mathrm{CDM}$ objects were matched to their DMAF simulation counterparts using TREEFROG and selecting the DMAF simulation halo with the largest merit function,

$M_{a b}=\frac{N_{a \cap b}^{2}}{N_{a} N_{b}}$,

where $N_{a}$ and $N_{b}$ are the number of particles in halos $a$ and $b$ while $\mathrm{N}_{a \cap b}$ is the number of uniquely identified particles that appear in both halos. In this work we restricted the merit function to counting only the dark matter particles due to the large depletion of gas in some of the simulations. For reference, we also show the best fit NFW density profile for the dark matter component scaled by

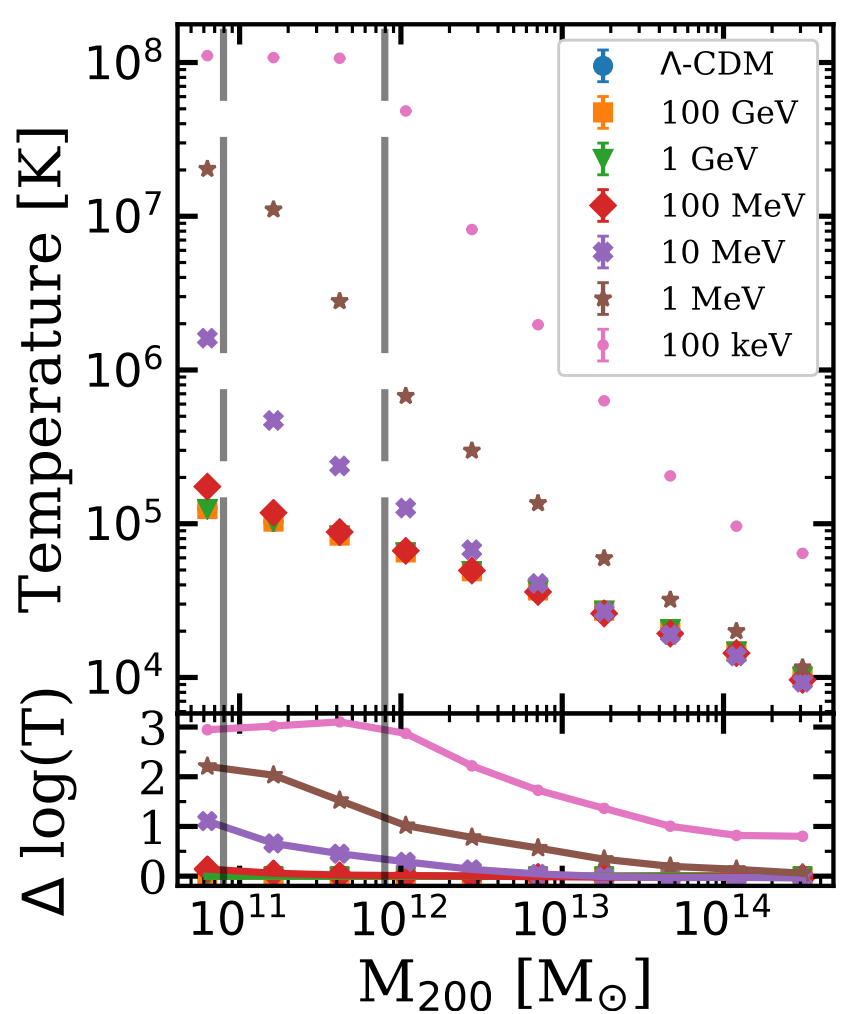

Figure 8. The average temperature of the halos gas as a function of the mass $\mathrm{M}_{200}$. We see that the $100 \mathrm{MeV}$ simulation is able to more strongly heat up the gas in less massive halos. The $100 \mathrm{keV}$ simulation has similarly heated up what little gas remains in their halos, see Figure 7.

$\Omega_{b} / \Omega_{\chi}$ (black profile) in the $\Lambda$ CDM simulation, but also show the dark matter density profiles in Figure 10 . The best fit line was calculated by minimising the equation

$\chi^{2}=\sum_{i=1}^{N} \frac{\rho\left(r_{i}\right)-\rho_{N F W}\left(r_{i} ; \rho_{0}, r_{s}\right)}{2 \sigma_{i}^{2}}$,

where $r_{i}$ are the logarithmically spaced radial bins, $\rho_{N F W}$ is given by equation 8 and $\rho\left(r_{i}\right)$ and $\sigma_{i}$ are the average and standard deviation of the density. The grey dashed vertical line shows average $\mathrm{R}_{200}$ scaled softening length $\epsilon_{\text {soft }}$ of the halos where we expect artificial gravitational softened cores to exist. In our fitting procedure we used 40 logarithmically spaced bins between $10 \epsilon_{\text {soft }}<\mathrm{r}$ $<\mathrm{R}_{200}$ so as not to include the artificial, gravitationally softened cores in the fit. A more careful analysis would also fit for the halo centres as a free parameter but here we simply centred the halos on the minimum potential.

The feedback has significantly altered the structure within the inner radius, $\left(<0.1 \mathrm{R}_{200}\right)$ of all samples with $m_{\chi}>1 \mathrm{GeV}$ DMAF and we see evidence that the density profiles are more sensitive as the average mass of the halo sample is decreased. In the $10^{12} \mathrm{M}_{\odot}$ sample, (bottom left panel), the $100 \mathrm{MeV}$ model shows a larger gas depletion of about 50 percent up to around $0.1 \mathrm{R}_{200}$. We also now see a small systematic depletion in the $1 \mathrm{GeV}$ model at this mass. For the smallest mass $10^{11} \mathrm{M}_{\odot}$ sub-sample we clearly see the 100 $\mathrm{MeV}$ model show stronger gas depletion now reaching out to the edge of the halos, as well as a larger systematic depletion in the $1 \mathrm{GeV}$ and even perhaps the $100 \mathrm{GeV}$ profile of about $10-20$ per- 
cent for radii greater than the softening. However the halos here are more poorly sampled due to small particle counts which affects the reliability of our centering, $\mathrm{R}_{200}$ estimates and density. Nonetheless this depletion appears in both the $1 \mathrm{GeV}$ and $100 \mathrm{GeV}$ model which were persistent even when a slightly different mass range was chosen. There is evidence that the dark matter profile has responded to changes in the gas density as seen in Figure 10. For the large cluster sized halos there is little change up to the softening lengths, but we see greater depletions of dark matter when the looking at smaller halos with stronger DMAF. This shows that weak DMAF could still alter the halo morphology for small halos without significantly altering the global statistics within a mass bin. For example, the $100 \mathrm{MeV}$ model shows clear depletion of gas in the inner regions of large galaxy sized objects $\left(10^{13} \mathrm{M}_{\odot}\right)$ but global statistics such as the HMF and gas fraction are not significantly affected at this range.

\section{CONCLUSIONS}

In this paper we have presented cosmological hydrodynamical simulations that contain Dark Matter Annihilation Feedback, in which sources of energy at places of high dark matter concentrations couple thermal energy into the nearby gas via interaction with their decay products. We have produced a set of $100 h^{-1} \mathrm{Mpc}$ wide box simulations with $\mathrm{N}=2 \times 512^{3}$ gas and dark matter particles from identical initial conditions and we examined the distribution of gas and dark matter particles as well as the properties of dark matter halos to explore differences generated by these DMAF events.

Our results from these simulations suggest two main conclusions and ways forwards with simulation studies of the DMAF. We find that small dwarf galaxies and sub-halos are sensitive to DMAF and would show direct evidence of feedback effects owing to their lower gravitational binding energies. In the $100 \mathrm{MeV}$ model for example, the abundance of halos is reduced by about 10 percent at redshift $z=0$ for halos with masses less than $10^{12} \mathrm{M}_{\odot}$. The evolution of the Halo Mass Function reveals that the mass scale below which this suppression occurs decreases with time, essentially allowing the HMF at high mass scales to progressively "catch up' with the $\Lambda \mathrm{CDM}$ halo abundance albeit at delayed time. In our simulations, the $100 \mathrm{MeV}$ case was able to catch up at late times $(z=0)$ for masses above $10^{12} \mathrm{M}_{\odot}$, but the delayed formation time due to the strong annihilation in the $100 \mathrm{keV}$ simulation results in almost no halos at any mass being able to catch up to their $\Lambda \mathrm{CDM}$ abundances at the end of the simulation, leaving about 50 percent less structure at all scales. Examination of our halos properties shows that while DMAF leads to higher temperature gas especially in lower mass halos, the dominant effect is to prevent gas condensation into the halos onto smaller dwarf halos and delaying the growth of the larger structures. The density profiles of our halos show that we expect weaker annihilation models to be able to alter the the morphology of the halos at smaller mass scales, where we see weak signs that even a $1 \mathrm{GeV}$ model can induce small changes at scales of $10^{11}-10^{12} \mathrm{M}_{\odot}$ in the inner regions of small halos.

As discussed in the introduction, models with dark matter particle masses less than $1 \mathrm{GeV}$ are met with tension from a number of astrophysical tests such as the CMB and Gamma Ray studies. This means the energy injection rates these models provide would only be relevant for exotic models with complicated decay spectra or behaviour that are somehow able to evade said constraints. For the models $\approx 1 \mathrm{GeV}$ or greater, this tension with observations is slackened such that they do not rule out even the simple S-wave annihilation WIMPs and our models at these scales show promise in imprinting signatures onto structures at dwarf halo mass scales below $\approx 10^{11} \mathrm{M}_{\odot}$.

Although the signs of DMAF are eventually washed out at high mass scales and late times - therefore harder to detect, the delay in the formation of these galactic structures could result in a difference of their star formation histories and ages thus allowing the possibility of detecting this type of dark sector physics even in the large structures.

\section{ACKNOWLEDGEMENTS}

The authors would like to acknowledge the University of Sydney HPC service for providing high-performance computational resources from ARTEMIS which has contributed to the research results reported within this paper as well as the assistance of resources and services from the National Computational Infrastructure (NCI), which is supported by the Australian Government. Nikolas Iwanus is supported by an Australian Postgraduate Award (APA). Florian List is supported by the University of Sydney International Scholarship (USydIS). We would like to thank the anonymous referee for their valued feedback which lead to improvements in the quality of this paper.

\section{REFERENCES}

Ackermann M., et al., 2014, Phys. Rev. D, 89, 042001

Ackermann M., et al., 2015, Phys. Rev. D, 91, 122002

Albert A., et al., 2017, ApJ, 834, 110

Arthur J., et al., 2017, MNRAS, 464, 2027

Ascasibar Y., 2007, A\&A, 462, L65

Barkana R., Loeb A., 1999, ApJ, 523, 54

Bartels R., Gaggero D., Weniger C., 2017, J. Cosmology Astropart. Phys., 5,001

Berlin A., Blinov N., 2018, Physical Review Letters, 120, 021801

Bertschinger E., 2006, Phys. Rev. D, 74, 063509

Binder T., Covi L., Kamada A., Murayama H., Takahashi T., Yoshida N., 2016, J. Cosmology Astropart. Phys., 11, 043

Boudaud M., Lavalle J., Salati P., 2017, Physical Review Letters, 119, 021103

Boylan-Kolchin M., Bullock J. S., Kaplinghat M., 2011, MNRAS, 415, L40

Bringmann T., Ihle H. T., Kersten J., Walia P., 2016, Phys. Rev. D, 94, 103529

Bullock J. S., Boylan-Kolchin M., 2017, ARA\&A, 55, 343

Bullock J. S., Kravtsov A. V., Weinberg D. H., 2000, ApJ, 539, 517

Bullock J. S., Dekel A., Kolatt T. S., Kravtsov A. V., Klypin A. A., Porciani C., Primack J. R., 2001, ApJ, 555, 240

Ceverino D., Klypin A., 2009, ApJ, 695, 292

Choquette J., Cline J. M., Cornell J. M., 2016, Phys. Rev. D, 94, 015018

Cui W., et al., 2016, MNRAS, 458, 4052

Diamanti R., Lopez-Honorez L., Mena O., Palomares-Ruiz S., Vincent A. C., 2014, J. Cosmology Astropart. Phys., 2, 017

Dooley G. A., Peter A. H. G., Vogelsberger M., Zavala J., Frebel A., 2016, MNRAS, 461, 710

Du X., Schwabe B., Niemeyer J. C., Bürger D., 2018, Phys. Rev. D, 97 , 063507

Durier F., Dalla Vecchia C., 2012, MNRAS, 419, 465

Elahi P. J., Thacker R. J., Widrow L. M., 2011, MNRAS, 418, 320

Elahi P. J., et al., 2013, MNRAS, 433, 1537

Elahi P. J., et al., 2016, MNRAS, 458, 1096

Elahi P. J., Welker C., Power C., Lagos C. d. P., Robotham A. S. G., Cañas R., Poulton R., 2018, MNRAS, 475, 5338

Elor G., Rodd N. L., Slatyer T. R., Xue W., 2016, J. Cosmology Astropart. Phys., 6, 024 


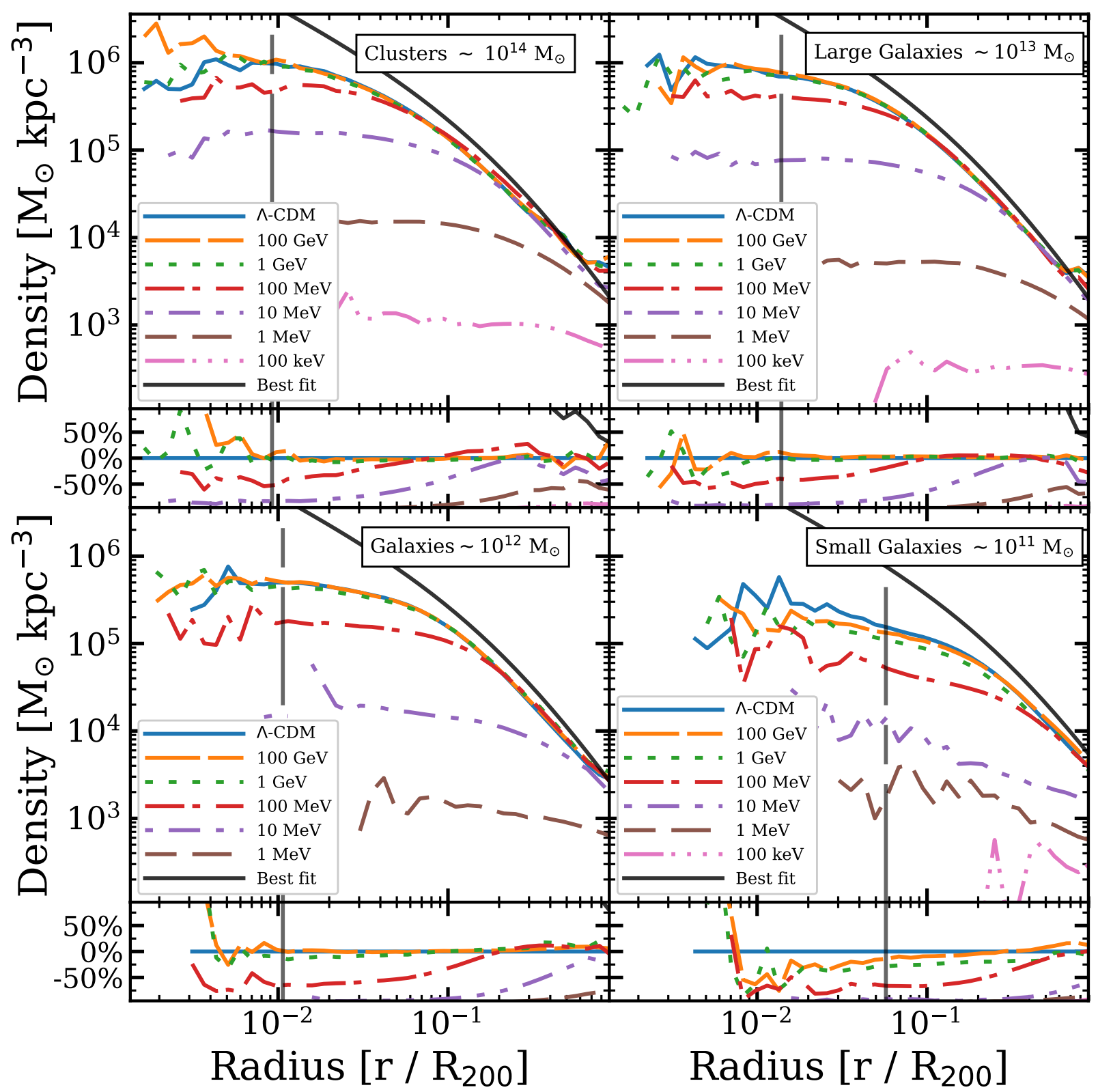

Figure 9. The averaged gas density profiles of 4 halo sub-samples with different masses. The residuals show the percentage density difference compared to the $\Lambda \mathrm{CDM}$ simulation. For the largest structures (top panels) the density on the out-skirts shows little difference for $100 \mathrm{MeV}$ dark matter particles but within $0.1 \mathrm{R}_{200}$ from the centre the gas has been severely depleted. The smaller structures (bottom panels) show gas depletion at all radii for the $100 \mathrm{MeV}$ case. At masses of about $10^{11} \mathrm{M}_{\odot}$ our averaged profiles become noisier as it becomes more difficult to achieve accurate centring and density estimates due to a smaller number of particles, but we see that there is a small but systematic deviation even above the typical softening length in the $100 \mathrm{GeV}$ case. The NFW density fit (dark line above) here has been scaled by $\frac{\Omega_{b}}{\Omega_{\chi}}$.

Fattahi A., Navarro J. F., Sawala T., Frenk C. S., Sales L. V., Oman K., Schaller M., Wang J., 2016, preprint, (arXiv:1607.06479)

Feng J. L., 2010, ARA\&A, 48, 495

Fitts A., et al., 2017, MNRAS, 471, 3547

Fry A. B., et al., 2015, MNRAS, 452, 1468

Griffen B. F., Ji A. P., Dooley G. A., Gómez F. A., Vogelsberger M., O'Shea B. W., Frebel A., 2016, ApJ, 818, 10

Grossi M., Springel V., 2009, MNRAS, 394, 1559

Heeck J., Teresi D., 2017, Phys. Rev. D, 96, 035018

Hoeft M., Yepes G., Gottlöber S., Springel V., 2006, MNRAS, 371, 401
Hopkins P. F., 2015, MNRAS, 450, 53

Hopkins P. F., 2017, arXiv e-prints, p. arXiv:1712.01294

Hopkins P. F., Quataert E., Murray N., 2012, MNRAS, 421, 3522

Hu W., Barkana R., Gruzinov A., 2000, Physical Review Letters, 85, 1158 Iwanus N., Elahi P. J., Lewis G. F., 2017, MNRAS, 472, 1214

Kamada A., Kaplinghat M., Pace A. B., Yu H.-B., 2017, Phys. Rev. Lett., 119,111102

Katz N., Gunn J. E., 1991, ApJ, 377, 365

Klypin A., Kravtsov A. V., Valenzuela O., Prada F., 1999, ApJ, 522, 82

Kravtsov A. V., Klypin A. A., Bullock J. S., Primack J. R., 1998, ApJ, 502, 


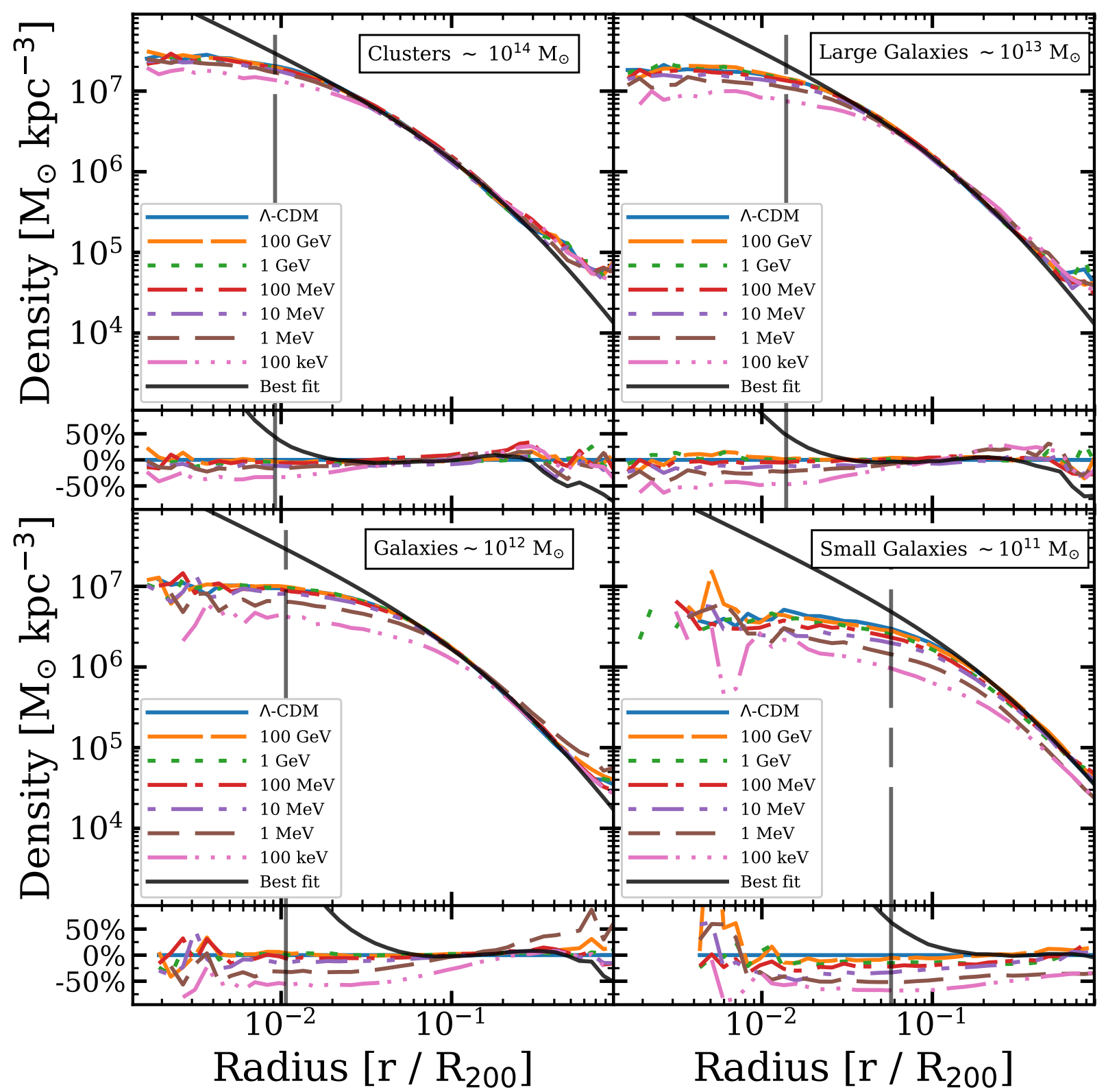

Figure 10. The sample averaged dark matter density profiles of 4 sub-samples of halos with different masses roughly corresponding to cluster, galaxy and dwarf sized halos. The residual panels show the percentage difference compared to the $\Lambda$ CDM simulation (as in Figure 9). Also shown is the best fit NFW profile to the $\Lambda \mathrm{CDM}$ simulation, see equation 8 . The greyed dashed line shows the average gravitational softening where the measured densities begin to diverge from the expected profile.

48

Krumholz M. R., Gnedin N. Y., 2011, ApJ, 729, 36

Leane R. K., Slatyer T. R., Beacom J. F., Ng K. C. Y., 2018, Phys. Rev. D, 98, 023016

Monaghan J. J., 1992, ARA\&A, 30, 543

Moore B., Ghigna S., Governato F., Lake G., Quinn T., Stadel J., Tozzi P., 1999, ApJ, 524, L19

Natarajan P., Croton D., Bertone G., 2008, MNRAS, 388, 1652

Navarro J. F., Frenk C. S., White S. D. M., 1996, ApJ, 462, 563

Nori M., Baldi M., 2018, MNRAS, 478, 3935

Oakes L., et al., 2017, International Cosmic Ray Conference, 301, 905

Oh S.-H., et al., 2015, AJ, 149, 180

Okoli C., 2017, preprint, (arXiv: 1711.05277)
Pappadopulo D., Ruderman J. T., Trevisan G., 2016, Phys. Rev. D, 94, 035005

Planck Collaboration et al., 2016, A\&A, 594, A13

Pontzen A., Governato F., 2012, MNRAS, 421, 3464

Power C., Navarro J. F., Jenkins A., Frenk C. S., White S. D. M., Springel V., Stadel J., Quinn T., 2003, MNRAS, 338, 14

Prada F., Klypin A. A., Cuesta A. J., Betancort-Rijo J. E., Primack J., 2012, MNRAS, 423, 3018

Profumo S., Queiroz F. S., Yaguna C. E., 2016, MNRAS, 461, 3976

Read J. I., Agertz O., Collins M. L. M., 2016, MNRAS, 459, 2573

Ren T., Kwa A., Kaplinghat M., Yu H.-B., 2018, preprint, (arXiv:1808.05695)

Rey-Raposo R., Dobbs C., Agertz O., Alig C., 2017, MNRAS, 464, 3536 
Rinchiuso L., Moulin E., Viana A., Van Eldik C., Johannes Veh for the H. E. S. S. collaboration 2017, preprint, (arXiv:1708.08358) Ripamonti E., Mapelli M., Ferrara A., 2007, MNRAS, 375, 1399

Robertson A., Harvey D., Massey R., Eke V., McCarthy I. G., Jauzac M., Li B., Schaye J., 2018, preprint, (arXiv:1810.05649)

Robles V. H., et al., 2017, MNRAS, 472, 2945

Saitoh T. R., Makino J., 2009, ApJ, 697, L99

Sawala T., et al., 2016, MNRAS, 457, 1931

Schön S., Mack K. J., Avram C. A., Wyithe J. S. B., Barberio E., 2015, MNRAS, 451, 2840

Schön S., Mack K. J., Wyithe J. S. B., 2018, MNRAS, 474, 3067

Sembolini F., et al., 2016a, MNRAS, 457, 4063

Sembolini F., et al., 2016b, MNRAS, 459, 2973

Somerville R. S., Davé R., 2015, ARA\&A, 53, 51

Spergel D. N., Steinhardt P. J., 2000, Physical Review Letters, 84, 3760

Springel V., 2015, NGenIC: Cosmological structure initial conditions, Astrophysics Source Code Library (ascl:1502.003)

Srisawat C., et al., 2013, MNRAS, 436, 150

Steigman G., Dasgupta B., Beacom J. F., 2012, Phys. Rev. D, 86, 023506

Thoul A. A., Weinberg D. H., 1996, ApJ, 465, 608

Tollet E., et al., 2016, MNRAS, 456, 3542

Tulin S., Yu H.-B., 2018, Phys. Rep., 730, 1

Valli M., Yu H.-B., 2018, Nature Astronomy, 2, 907

Veltmaat J., Niemeyer J. C., 2016, Phys. Rev. D, 94, 123523

Veltmaat J., Niemeyer J. C., Schwabe B., 2018, Phys. Rev. D, 98, 043509

Vogelsberger M., Zavala J., Simpson C., Jenkins A., 2014, MNRAS, 444, 3684

Wechakama M., Ascasibar Y., 2011, MNRAS, 413, 1991

Wetzel A. R., Hopkins P. F., Kim J.-h., Faucher-Giguère C.-A., Kereš D., Quataert E., 2016, ApJ, 827, L23

Xiang Q.-F., Bi X.-J., Lin S.-J., Yin P.-F., 2017, Physics Letters B, 773, 448

Zhao Y., Bi X.-J., Jia H.-Y., Yin P.-F., Zhu F.-R., 2016, Phys. Rev. D, 93, 083513

Zhao Y., Bi X.-J., Yin P.-F., Zhang X., 2018, Phys. Rev. D, 97, 063013

van den Aarssen L. G., Bringmann T., Pfrommer C., 2012, Physical Review Letters, 109, 231301

\section{APPENDIX A: ORIGIN OF THE HOT BUBBLES}

In simulations with high injection rates, we see the formation of hot bubbles; a $100 \mathrm{keV}$ simulation, see first panel Figure A1, shows the formation of these bubbles at all resolutions while the $10 \mathrm{MeV}$ simulation only formed these bubbles in the $2 \times 512^{3}$ simulation. The bubbles appear even with stricter time-stepping and global time steps, see the middle panel Figure A1, suggesting they are not simply a result of insufficient time-step limiters such in Saitoh \& Makino (2009) or Durier \& Dalla Vecchia (2012), rather they are due to our assumption that the coupling to the gas is always 100 percent efficient and local. These bubbles originate from areas of the simulation where the gas is depleted relative to the dark matter density (like in the smaller halos). In these areas, the gas receives the same amount of energy but now shared shared amongst less dense gas leading to further rarefaction of a hotter gas which further expands the bubble, this trend continues as the heating is never shut off.

We have performed a simple check to show that this is the case by implementing a gas efficiency step function. If a gas particle reaches a density lower than the cosmic average, e.g. $\alpha_{c u t}$ $\Omega_{b}>\frac{\rho_{i}}{\rho_{\text {crit }}}$ then we consider the gas too low density to absorb the products efficiently and it receives no further energy. We have run this on smaller sized simulations with a resolution of $2 \times 128^{3}$ particles; see Figure A1 bottom panel for the simulation run with $\alpha_{c u t}=0.8$. Although these low density regions still form we found that this density cut off heavily suppresses the formation and

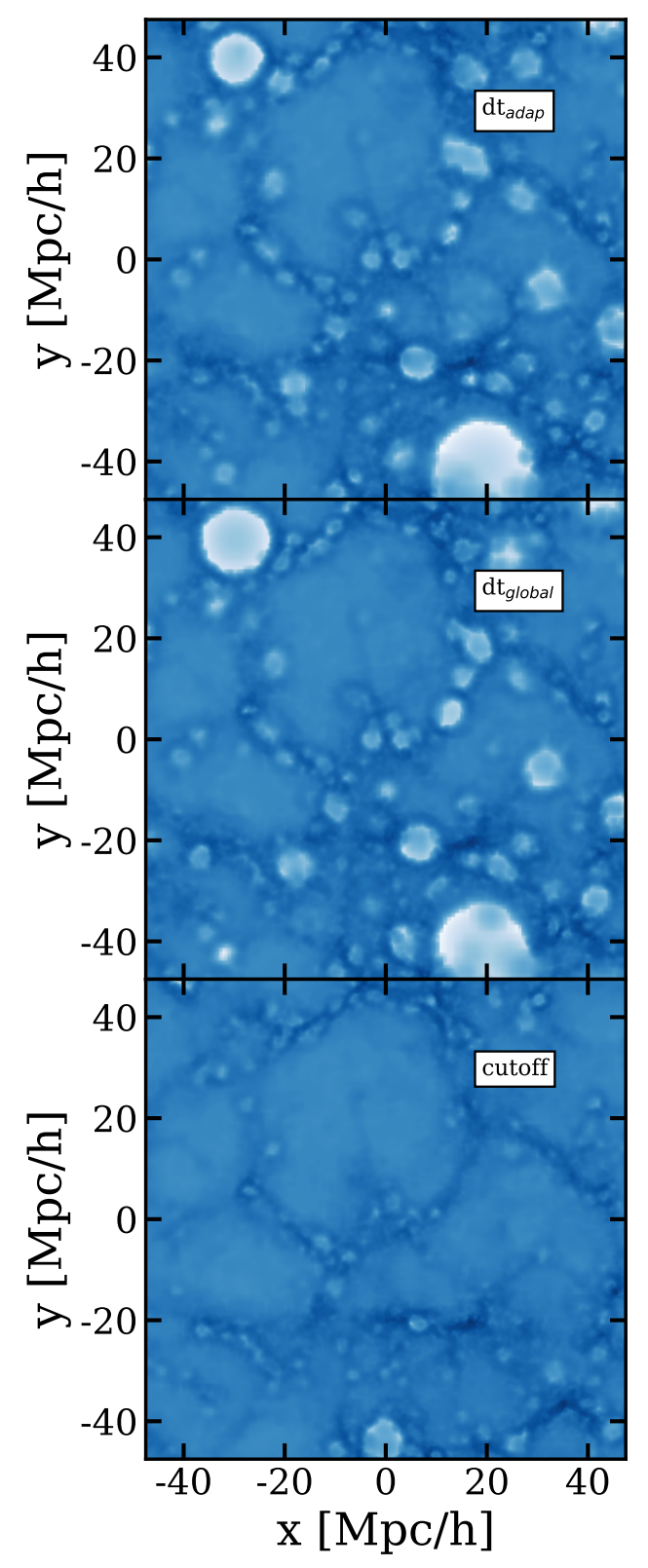

Figure A1. The gas density of three $100 \mathrm{keV}$ simulations with different time stepping schemes. They contain $\mathrm{N}=2 \times 128^{3}$ particles with the regular GADGET-2 adaptive time-stepping scheme ( $\left.\mathrm{dt}_{\text {ad ap }}\right)$, global equal time steps ( $\mathrm{dt}_{\text {equal }}$ ). In addition to a simulation run with a heating cutoff at low densities $\left(\frac{\rho_{\text {cut }}}{\rho \text { crit }}=0.8 \Omega_{b}\right)$ to suppress the growth and formation of bubbles that form in areas where the gas density is reduced strongly.

growth of these bubbles. We retrieve the same results as expected from lower resolutions of a largely homogeneous gas with no or few gas particles bound to structure. The density phase space distribution is only strongly affects high temperature low-density particles; see Figure A2. We have also checked that the other results in this work, such as the halo mass functions, baryon fractions and temperatures and found sub-percent differences despite the stricter 

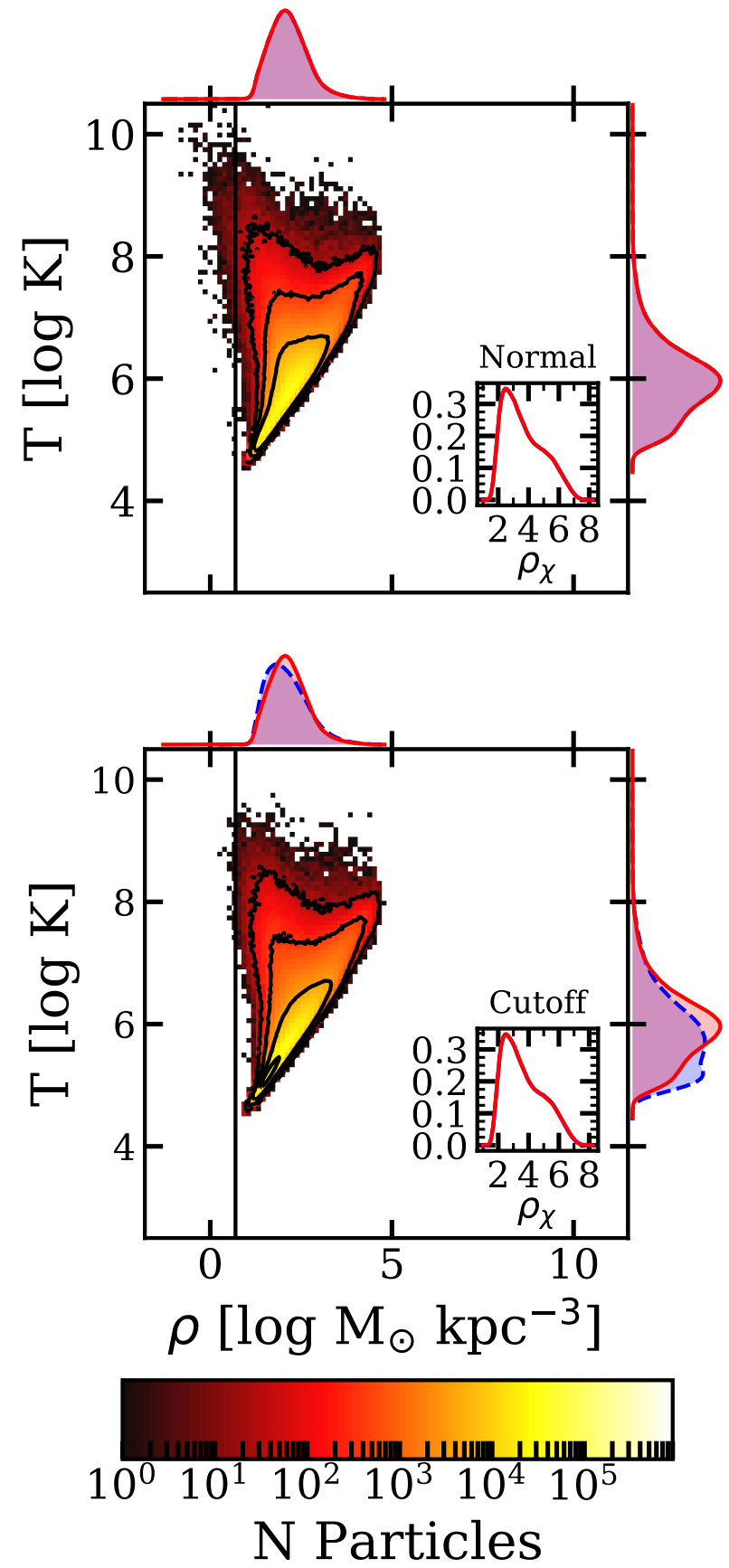

Figure A2. Comparison of two $100 \mathrm{keV}$ simulations, with $\mathrm{N}=2 \times 128^{3}$ particles with the normal GADGET-2 adaptive time-stepping scheme as well as a simulation run with equal time-steps in addition to a heating cutoff switch at low densities $\left(\frac{\rho_{\text {cut }}}{\rho \text { crit }}=0.8 \Omega_{b}\right)$ shown as black vertical line, to suppress the formation of bubbles. The $2 \mathrm{D}$ distribution largely retains the same overall shape although we have reduced the amount of hot low density gas particles below the density cutoff.

time stepping and percent level differences with the cutoff, smaller then the error of the binning ${ }^{4}$.

These bubbles only occur when the gas is sufficiently depleted in the dark matter structure anyway. For example the baryon fraction of our halos is unchanged when the bubbles are suppressed because VELOCIRAPTOR considers these gas particles to unbound to the halos anyway. The halo mass functions are also unaffected because none of the rarefied gas is considered bound and so has little gravitational influence on the structures, other than not being accreted in the first place. For this reason we believe that bubbles we see in Figure A2 have minimal impact, the results presented in the rest of the paper are sound; the sharp density contrasts only exist in an extremely rarefied and unbound gas. In addition, for testing purposes we have also implemented DMAF into the more modern code GIZMO which contains time-stepping limiters as in Durier \& Dalla Vecchia (2012) as well as the pressure formulation of SPH, meshless finite-volume and mass hydrodynamic implementations, see Hopkins $(2015,2017)$ and find that the bubbles arise in all configurations, although this GIZMO implementation is less thoroughly tested than our GADGET-2 implementation. Investigations are currently underway systematically studying the detailed effects due to different hydrodynamic implementations with DMAF and are anticipated in the next paper of this series.

The bubbles may be diminished with a distributed energy injection scheme and non-localised heating. As the gas density falls off we expect the injected energy to be more spread around out rather than concentrating in these hot, low density bubbles and we aim to study the result of energy injection dispersed over a mean free path in future work. Our simulations are also non-cooling and so would also be aided by thermal conduction, radiative cooling etc. which are likely to help prevent such an extreme build up. These changes are anticipated in future codes but for now these bubbles are only prominent in the strong injection models anyway and do not significantly affect our results within the halos themselves.

This paper has been typeset from a $\mathrm{T}_{\mathrm{E}} \mathrm{X} / \mathrm{L} \mathrm{T}_{\mathrm{E}} \mathrm{X}$ file prepared by the author.

\footnotetext{
${ }^{4}$ We tried a range of cutoffs between 0.3-0.8. Lower cutoffs led to less suppression of the bubbles, but smaller differences.
} 